sporadic in origin. The cause of sporadic AD is likely to be multifactorial, with external factors interacting with biological or genetic susceptibilities to accelerate the manifestation of the disease. Diabetes mellitus (DM) might be such factor, as there is extensive data from epidemiological studies suggesting that DM is associated with an increased relative risk for Alzheimer's disease. Type 1 diabetes (T1DM) and type 2 diabetes (T2DM) are known to affect multiple cognitive functions in patients. However, the consequences of both type of diabetes on tau pathology are not well understood. Methods: We wanted to investigate whether T1DM and T2DM induce tau pathology in different models of diabetes. For T1DM, we used a genetic model (Non Obese Diabetic), or we induced it with streptozotocin. For T2DM we used a genetic model (db/db mice), or we induced it with high fat diet. For all these models we investigated tau pathology and its mechanism. Results: We observed tau hyperphosphorylation in all the forms of diabetes. However, the mechanism of tau phosphorylation was different between T1DM and T2DM. Conclusions: Our data indicate that: i) diabetes induced abnormal tau hyperphosphorylation in the brain of normal mice, with biochemical, regional and histochemical patterns resembling those in early $\mathrm{AD}$ brains. ii) diabetes induced tau hyperphosphorylation through two distinct mechanisms between T1DM and T2DM. This research will help the development of treatments or life-style strategies destined to check the advance of the disease.

\section{P1-376 A-BETA 42 AND A-BETA 40 FIBRILLATION, IN VITRO, IS AFFECTED TO DIFFERENT EXTENTS BY ENVIRONMENTAL STRESS FACTORS}

\author{
Atta Ahmad ${ }^{1,2},{ }^{1}$ University of Michigan, Ann Arbor, MI, USA; \\ ${ }^{2}$ Massachusetts Institute of Technology, Cambridge, MA, USA. \\ Contacte-mail:ahmadatt@umich.edu
}

Background: Alzheimer's disease (AD) in humans is a common progressive neurodegenerative disease, associated with cognitive dysfunction, memory loss and neuronal loss that affects millions of old age people worldwide. Alzheimer peptides $A B 40$ and $A B 42$ are precursors of the neurotoxic oligomers, protofibrils and amyloid fibers that accumulate in the brains of these patients. The extent of the involvement of $\mathrm{A} \beta 42$ and $\mathrm{A} \beta 420$ on $\mathrm{AD}$ is not well understood and some differences have been published. Methods: Here we report SEC, CD, ThT, MTT, EM data that further leads to characterizing their similarities and differences. Results: Besides the basic differences in the kinetics of fibrillation of the two peptides, they respond differently to the aggregation inducing factors. We observed calcium selectively enhanced fibrillation of $A B 42$ and did not affect the kinetics of $A B 40$. AB42 populated abundant intermediate aggregated species while $\mathrm{A} \beta 42$ intermediates were scarce and different from those of A 342 . Both peptides exhibited different aggregation kinetics in DMEM. Conclusions: Analyzing data with respect to other published work in this area we conclude that the two extra residues in $\mathrm{A} \beta 42$ are the source of observed differences.

\section{P1-377 AMYLOID PET IN MILD COGNITIVE IMPAIRMENT AND ALZHEIMER'S DISEASE WITH [ $\left.{ }^{11} \mathrm{C}\right]$ BF-227: COMPARISON TO VOXEL-BASED MORPHOMETRIC MRI}

Masaaki Waragai ${ }^{1}$, Nobuyuki Okamura ${ }^{2}$, Katsutoshi Furukawa ${ }^{1}$, $\mathrm{He} \mathrm{Shao}^{3}$, Manabu Tashiro ${ }^{4}$, Shozo Furumoto ${ }^{5}$, Kentaro Sugi ${ }^{3}$, Kazuhiko Yanai ${ }^{2}$, Yukitsuka Kudo ${ }^{6}$, Hiroyuki Arai ${ }^{1},{ }^{1}$ Department of Geriatrics and Gerontology, Division of Brain Sciences, Institute of Development, Aging and Cancer, Tohoku University, Sendai, Japan; ${ }^{2}$ Department of Pharmacology, Tohoku University School of Medicine, Sendai, Japan; ${ }^{3}$ Department of Pharmacology, Tohoku University School of Medicine, Sendai, Japan; ${ }^{4}$ Division of Cyclotron Nuclear Medicine, Cyclotron and Radioisotope Center, Tohoku University, Sendai, Japan; ${ }^{5}$ Department of Nuclear Medicine and Radiology, Institute of Development, Aging and Cancer, Tohoku University, Sendai, Japan; ${ }^{6}$ Innovation of New Biomedical Engineering Center, Tohoku University, Sendai, Japan.

Contacte-mail:waragai@kk.iij4u.or.jp

Background: The beta amyloid (A $\beta$ ) plaque is an important pathological hallmark of Alzheimer's disease (AD). Although still controversial, $\mathrm{A} \beta$ de- position is believed to be directly associated with neuronal loss, cognitive dysfunction, and resultant cerebral atrophy. To evaluate the clinical usefulness of positron emission tomography (PET) imaging using $\left[{ }^{11} \mathrm{C}\right] \mathrm{BF}-227$ as a quantitative marker of $\mathrm{A} \beta$ burden and voxel-based morphometry analysis of MRI (VBM-MRI) for early diagnosis and tracking of the severity of AD. Methods: The amyloid plaque burden and grey matter volume were evaluated using neocortical BF-227 retention and VBM-MRI respectively, in 14 aged normal controls, 18 subjects with mild cognitive impairment (MCI), and $20 \mathrm{AD}$ patients. All MCI patients were followed at least 2 years and divided into two groups, the MCI converters who developed to $\mathrm{AD}$ and the non-converters. Results: Both MCI and AD subjects exhibited significant elevation of BF-227 retention and parahippocampal gray matter loss on MRI. Receiver operating characteristic analysis revealed that BF-227PET distinguishes between subjects with $\mathrm{AD}$ and the normal population with higher sensitivity and specificity than MRI analysis. The MCI converters to $\mathrm{AD}$ were more clearly distinguished from the $\mathrm{MCI}$ non-converters using BF-227 PET than using VBM-MRI. A significant correlation with cognitive function in $\mathrm{AD}$ patients was observed for global gray matter loss in MRI but not neocortical retention of BF-227. Conclusions: These findings suggest that measurement of $\mathrm{A} \beta$ burden using BF-227-PET is better suited for early diagnosis of AD than VBM-MRI. However, evaluation of $\mathrm{A} \beta$ burden alone might be insufficient for tracking of the severity of dementia, and VBM-MRI appears to be better suited for this purpose than amyloid PET.

\section{P1-378 NEUROSTRUCTURAL PREDICTORS OF ALZHEIMER'S DISEASE: A META-ANALYSIS OF VBM STUDIES}

Luiz K. Ferreira ${ }^{1}$, Breno S. Diniz ${ }^{2}$, Orestes V. Forlenza ${ }^{2}$, Geraldo F. Busatto ${ }^{1}$, Marcus V. Zanetti ${ }^{1},{ }^{1}$ Laboratory of Psychiatric Neuroimaging (LIM-21), Department and Institute of Psychiatry, Faculty of Medicine, University of São Paulo, São Paulo, Brazil; ${ }^{2}$ Laboratory of Neurosciences (LIM-27), Department and Institute of Psychiatry, Faculty of Medicine, University of São Paulo, São Paulo, Brazil.

Contacte-mail: kobuti@yahoo.com

Background: The identification of biological markers at early stages of Alzheimer's disease $(\mathrm{AD})$ contributes to diagnostic accuracy and adds prognostic value. Early diagnosis is very important as treatment and preventive interventions will be more efficacious if started at pre-dementia stages. Structural magnetic resonance imaging (MRI) is a safe and widely available method and is a promising tool to improve accuracy of early diagnosis. However, in spite of recent developments, results of neurostructural imaging studies on predicting conversion to $\mathrm{AD}$ are not uniform. We aimed to conduct a systematic review and meta-analysis of voxel-based morphometry (VBM) studies about the neurostructural predictors of conversion to AD. Methods: We carried out a systematic and comprehensive search in September 18, 2009 using Medline, ISI Web of Science, PsycInfo, Embase and Scopus databases for longitudinal VBM studies investigating conversion to $\mathrm{AD}$. We selected studies that used whole brain VBM methods to compare converters and non-converters according to baseline brain MRI. Meta-analysis was based on the activation likelihood estimation method using the revised algorithm in Ginger Ale 2.0 program. This method aims to indicate the anatomical convergence between results from different studies and requires the

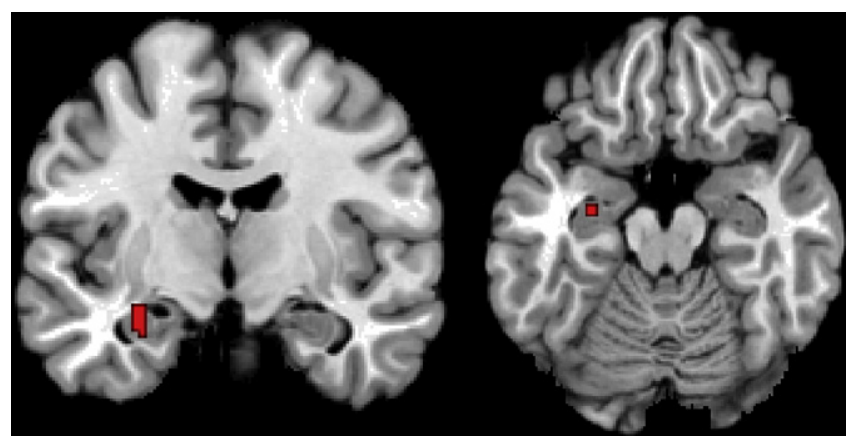

